\title{
EC Radiative Transport and Losses in DEMO-like High-Temperature Plasmas
}

\author{
F. Albajar, ${ }^{1, a}$, M. Bornatici ${ }^{2}$, and F. Engelmann ${ }^{3}$ \\ ${ }^{1}$ Fusion for Energy, Josep Pla 2, Barcelona, 08019, Spain \\ ${ }^{2}$ Physics Department, University of Pavia, Pavia, 27100, Italy \\ ${ }^{3}$ Max-Planck-Institut für Plasmaphysik, Garching, 85748, Germany
}

\begin{abstract}
Electron cyclotron (EC) wave power losses tend to play a significant role in the local electron power balance of a tokamak fusion reactor for electron temperatures above $35 \mathrm{keV}$ as considered for steadystate operation. Using the RAYTEC code a comparative study of EC wave and bremsstrahlung contributions to the local and total power balance in relation to the $\alpha$-particle heating power was carried out for a range of plasma conditions, wall reflection coefficients of the EC waves, and magnetic fields.
\end{abstract}

\section{Introduction}

In view of the potential benefits of operating DEMO at high plasma temperature [1], electron cyclotron (EC) radiative transport and losses are of particular relevance since in this case the locally radiated net EC power density in the plasma core tends to become significant with respect to the power density of fusion $\alpha$-particle heating [2,3]. To cover $\alpha$-particle heating the RAYTEC code [4] was complemented with Brunelli's fit [5] for the DT fusion cross-section,

$$
<\sigma v>=9 \times 10^{-22} \exp \left[-0.476|\ln (T / 69)|^{2.25}\right]\left(\mathrm{m}^{3} \mathrm{~s}^{-1}\right),
$$

which provides an excellent approximation up to electron temperatures of $150 \mathrm{keV}$.

Here, a detailed analysis of the net EC radiation losses in high-temperature DEMO-like plasmas is presented. For comparison, bremsstrahlung losses as present in an impurity-free plasma are also calculated. Parametric studies of the radial profile of the EC wave radiation losses as well as of the total power loss have been carried out in the electron temperature (central values of $T_{\mathrm{e} 0}=40$, 60 and $80 \mathrm{keV}$ and an "advanced" profile [4]) combined with different choices of the plasma electron density and two electron density profiles (a flat [4] and a peaked one as suggested in Ref.[6]), in the (average) wall reflection coefficient of the EC waves $\left(R_{\mathrm{w}}=0.6,0.7\right.$ and 0.8$)$, and in the toroidal magnetic field (values on axis $B_{\mathrm{t} 0}=6,8$, $10 \mathrm{~T}$ ). The results are put in relation to the corresponding bremsstrahlung losses and to $\alpha$-particle heating (assuming no dilution effect).

\footnotetext{
a Corresponding author: Ferran.albajar@f4e.europa.eu
}

Table 1. Main parameters of reference cases. For further use, the total radiative power losses via $\mathrm{EC}$ waves $\left(P_{\mathrm{EC}}\right)$ and bremsstrahlung $\left(P_{\text {brems }}\right)$ as calculate with RAYTEC are also given.

\begin{tabular}{|c|c|c|c|}
\hline Case & $\begin{array}{c}\text { A: Flat } \\
\text { density } \\
\text { profile at } \\
\text { fixed } \\
P_{\alpha}=600 \mathrm{MW} \\
\left(T_{e 0}=60 \mathrm{keV}\right)\end{array}$ & $\begin{array}{c}\text { B: Peaked } \\
\text { density } \\
\text { profile at } \\
\text { fixed } \\
P_{\alpha}=600 \mathrm{MW} \\
\left(T_{e 0}=60 \mathrm{keV}\right)\end{array}$ & $\begin{array}{c}\text { C: Peaked } \\
\text { density } \\
\text { profile at } \\
\text { fixed } \\
P_{\alpha}=600 \mathrm{MW} \\
\left(T_{e 0}=40 \mathrm{keV}\right)\end{array}$ \\
\hline $\begin{array}{c}n_{\mathrm{e} 0} \\
\left(10^{19} \mathrm{~m}^{-3}\right)\end{array}$ & 7.19 & 8.24 & 9.00 \\
\hline $\begin{array}{c}\alpha_{\mathrm{n}} / n_{\mathrm{edge}} \\
\left(10^{19} \mathrm{~m}^{-3}\right)\end{array}$ & $0.1 / 0$ & $1.4 / 4$ & $1.4 / 5$ \\
\hline $\begin{array}{c}<n_{e}> \\
\left(10^{19} \mathrm{~m}^{-3}\right)\end{array}$ & 6.54 & 5.77 & 6.67 \\
\hline $\begin{array}{c}T_{\mathrm{e} 0} \\
(\mathrm{keV})\end{array}$ & 60 & 60 & 40 \\
\hline $\begin{array}{c}<T_{\mathrm{e}}> \\
(\mathrm{keV})\end{array}$ & 23.96 & 23.96 & 15.98 \\
\hline$<\beta \mathrm{t}>$ & 0.037 & 0.037 & 0.028 \\
\hline $\begin{array}{c}P_{\alpha} \\
(\mathrm{MW})\end{array}$ & 600 & 600 & 600 \\
\hline $\begin{array}{c}P_{\mathrm{EC}} \\
(\mathrm{MW})\end{array}$ & 213 & 218 & 77 \\
\hline $\begin{array}{c}P_{\mathrm{brems}} \\
(\mathrm{MW})\end{array}$ & 37 & 36 & 37 \\
\hline
\end{tabular}

The basic DEMO-like tokamak plasma parameters adopted are: plasma major and minor radius, respectively, $R=8.5 \mathrm{~m}$ and $a=2.742 \mathrm{~m}$ (aspect ratio $A=3.1$ ); toroidal magnetic field on axis $B_{\mathrm{t} 0}=6 \mathrm{~T}$, and vertical elongation $\kappa=1.7$. Specifically, the plasma profiles are of the form 


$$
X(\rho)=X(1)+[X(0)-X(1)]\left(1-\rho^{\beta_{x}}\right)^{\alpha_{x}},
$$

where $\rho$ is the normalized radial coordinate $r / a$. For the "advanced" temperature profile $\beta_{T}=5.4$ and $\alpha_{T}=8.0$ is chosen [4]. The two density profiles considered are characterised by $\beta_{n}=2$ and, respectively, $\alpha_{n}=0.1$ and $\alpha_{n}=1.4$ for the flat [4] and the peaked profile [6]. The fusion power generated is taken to be $3 \mathrm{GW}$ so that the $\alpha$ particle heating power amounts to $P_{\alpha}=600 \mathrm{MW}$. The main parameters of the three reference cases are listed in Table 1, the related electron temperature and density profiles are shown in Fig. 1. The reference value taken for the wall reflection coefficient is $R_{\mathrm{w}}=0.8$; this comparatively high value implies that the corresponding $\mathrm{EC}$ wave power losses result to be rather on the low side.
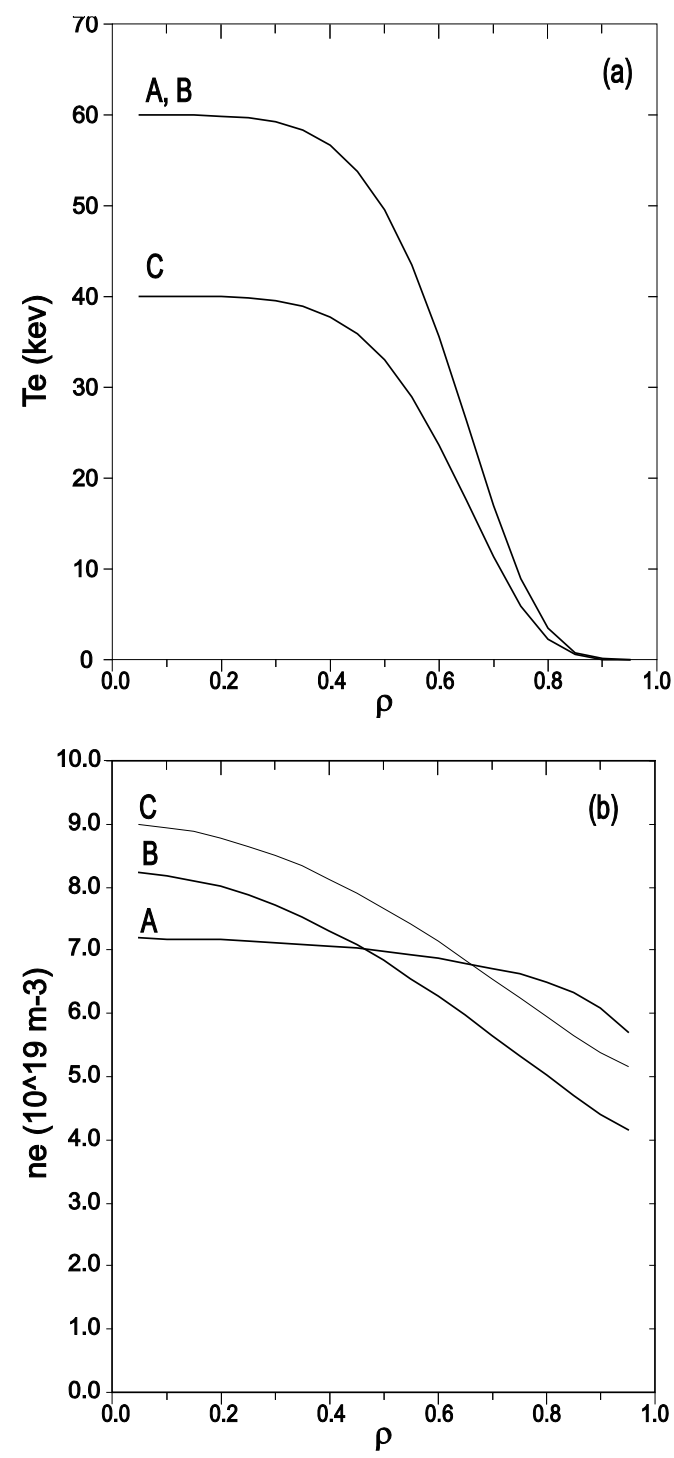

Figure 1. Profiles of the electron temperature (a) and density (b) of the three reference cases of Table 1 in terms of the normalised radial coordinate $\rho$.

\section{Results}

\subsection{Radial profiles of power losses for the reference cases}

The radial profiles of the radiative power losses and of $\alpha$ particle heating as calculated with RAYTEC are shown in Fig.2. Both the figure and Table 1 corroborate that EC wave losses, even for a wall reflection coefficient as high as 0.8 , are significant for a peak electron temperature of $40 \mathrm{keV}$ and become an essential loss channel for still higher temperatures. At a peak electron temperature of 60 $\mathrm{keV}$, for a flat density profile, the $\mathrm{EC}$ waves exhaust about $35 \%$ of the $\alpha$-particle heating power, and in the hot core their contribution to the power balance is about $60 \%$ of the local $\alpha$-particle heating power density. In all cases, losses by bremsstrahlung tend to be less important than EC wave losses. Comparison of cases A and B shows that the form of the density profile has only minor influence. The non-monotonous profile of the net EC wave power loss as characteristic for plasmas with a wide hot core optically thick for the low-frequency EC wave spectrum, is recovered in all cases.

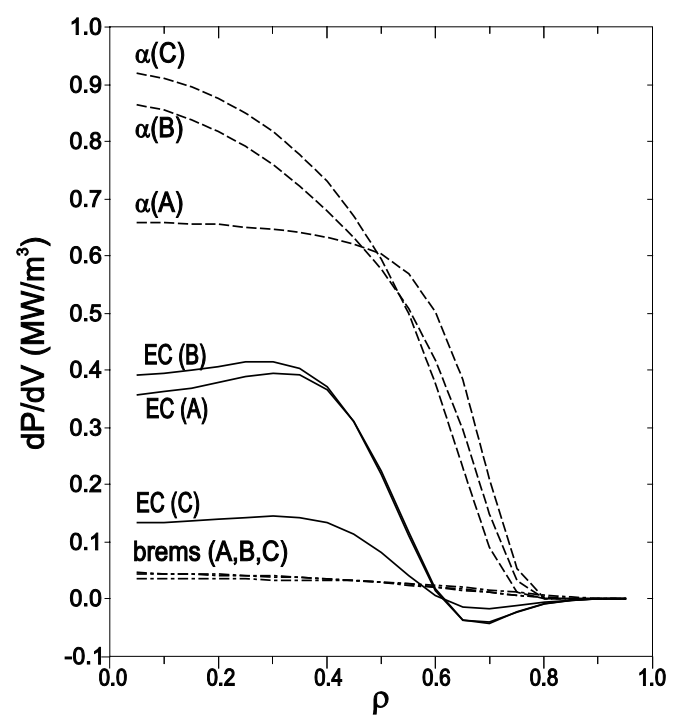

Figure 2. Radial profiles of radiative power losses through EC waves and bremsstrahlung for the reference cases of Table 1; for comparison, the $\alpha$-particle heating power density is also shown.

\subsection{Variation of electron temperature for flat density}

Starting from the reference case A, a systematic study of the impact of the electron temperature was carried out. In all cases, the fusion power is kept at $3 \mathrm{GW}$; to achieve this, the density and, correspondingly, the value of $\langle\beta \mathrm{t}\rangle$ is adjusted while all other parameters are the same as for case A (see Table 1). Fig. 3 shows, along with case A, the results for peak temperatures of 40 and $80 \mathrm{keV}$, the related peak densities being, respectively, $n_{\mathrm{e} 0}=8.13 \times 10^{19}$ and $6.86 \times 10^{19} \mathrm{~m}^{-3}$.

Again it is seen that bremsstrahlung losses are comparatively unimportant, the more the higher the electron temperature is. In fact, they result to be close to temperature-independent because for fixed fusion power the effect of increasing the temperature tends to be compensated by the reduction of density. On the other hand, for peak electron temperatures in the range of 60 $\mathrm{keV}$ and higher, in the hot core, EC wave losses tend to 
become the dominant $\alpha$-particle power exhaust channel. For $T_{\mathrm{e} 0}=80 \mathrm{keV}$, they there even exceed the $\alpha$-particle heating power so that the adopted plasma conditions cannot be a steady state. The total EC wave power losses calculated using RAYTEC are found to be well approximated by those derived from the analytic model based on using the locally applied Trubnikov formula (LATF) as considered in Ref.[4] (this does not apply in the same way to EC power density profiles obtained from LATF [4]). Also, it is worthwhile to note that the global scaling of the total EC wave power loss given by Trubnikov [7], $P_{\mathrm{EC}} \sim n_{\mathrm{e}}{ }^{0.5} T_{\mathrm{e}}^{2.5}$, describes the RAYTEC results, in the range considered, with an accuracy better than $10 \%$.

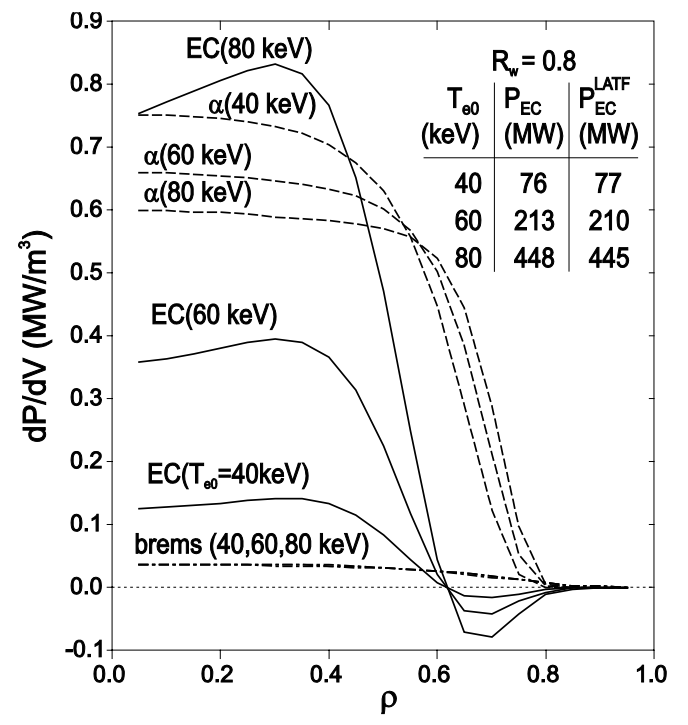

Figure 3. Radial profiles of radiative power losses through EC waves and bremsstrahlung and of $\alpha$-particle heating as well as, in the insert, the total EC wave power loss $P_{\mathrm{EC}}$ from RAYTEC calculations and from LATF for the reference case A of Table 1 and for peak electron temperatures of 40 and $80 \mathrm{keV}$ at fixed fusion power.

\subsection{Variation of the wall reflection coefficient}

To quantify the impact of lower wall reflection, for the plasma conditions of reference case A the profile of the net radiated EC wave power and the total EC power loss $P_{\mathrm{EC}}$ were determined for wall reflection coefficients of $R_{\mathrm{w}}=0.7$ and 0.6. For $R_{\mathrm{w}}=0.6$, both the EC wave power radiated from the hot core and the total EC power loss are about $30 \%$ higher than for $R_{\mathrm{w}}=0.8$ (see Fig. 4). This increase is owing to the reduction of the effective optical thickness of the system when wall reflection is less strong. The RAYTEC results follow the scaling $P_{\mathrm{EC}} \sim\left(1-R_{\mathrm{w}}\right)^{0.5}$ due to Trubnikov [7] with an accuracy of $1 \%$ in the parameter range considered.

\subsection{Variation of the confining toroidal field}

In order to display the impact of higher confining magnetic fields, sometimes also under consideration for DEMO, the parameter sets of Table 2 have been analysed. The cases of $B_{\mathrm{t} 0}=8$ and $10 \mathrm{~T}$ have been constructed from the reference case A, keeping the geometry, wall reflection properties, fusion power, electron temperature and profiles, as well as $\langle\beta \mathrm{t}\rangle$ unchanged. This implies that the electron density is scaled like $B_{\mathrm{t} 0}{ }^{2}$; as a consequence, the power densities of $\alpha$-particle heating and bremsstrahlung loss which scale in the same way (cf. Fig. 5), do scale like $B_{\mathrm{t} 0}{ }^{4}$, and the plasma radii have to be scaled like $B_{\mathrm{t} 0}{ }^{-4 / 3}$. The total power loss due to bremsstrahlung is unchanged.

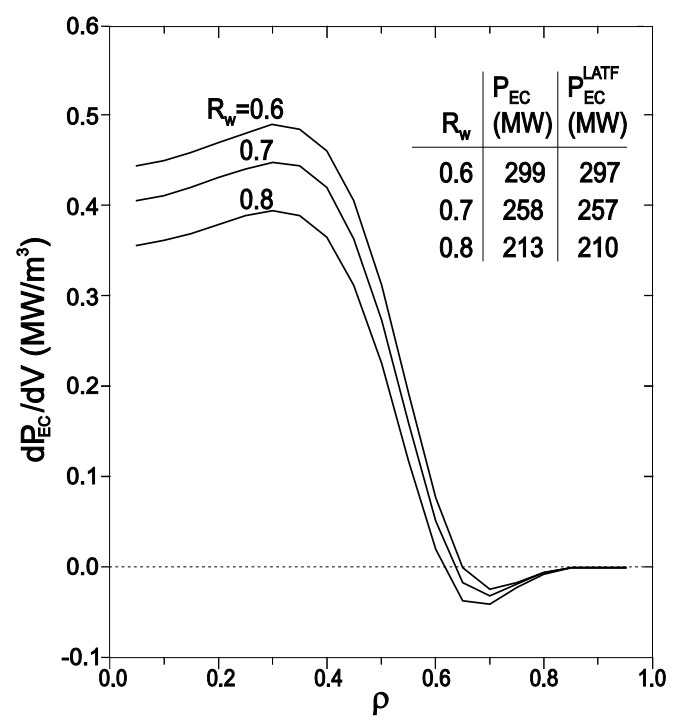

Figure 4. Radial profiles of net radiative EC wave power losses as calculated with RAYTEC for wall reflection coefficients of 0.6 and 0.7 compared to case $\mathrm{A}(\mathrm{Rw}=0.8)$. The corresponding total EC power losses, along with LATF results, are given in the insert.

Table 2. Plasma parameters adopted for the high magnetic field study along with those of reference case A, as well as total EC wave $\left(P_{\mathrm{EC}}\right)$ and bremsstrahlung $\left(P_{\text {brems }}\right)$ power losses as computed by RAYTEC; the EC wave power loss normalised to the total $\alpha$-particle heating power $P_{\alpha}$ and the average radiative power flux onto the first wall, $F_{\text {rad }}$, are also given.

\begin{tabular}{|c|c|c|c|}
\hline$B_{\mathrm{t} 0}(\mathrm{~T})$ & 6 (case $\mathrm{A})$ & 8 & 10 \\
\hline$R$ & 8.5 & 5.8 & 4.3 \\
\hline$a$ & 2.742 & 1.871 & 1.387 \\
\hline$T_{\mathrm{e} 0}(\mathrm{keV})$ & 60 & 60 & 60 \\
\hline$<T_{\mathrm{e}}>(\mathrm{keV})$ & 23.96 & 23.96 & 23.96 \\
\hline$n_{\mathrm{e} 0}\left(10^{19} \mathrm{~m}^{-3}\right)$ & 7.19 & 12.76 & 19.98 \\
\hline$<n_{\mathrm{e}}>\left(10^{19} \mathrm{~m}^{-3}\right)$ & 6.54 & 11.60 & 18.17 \\
\hline$<\beta_{\mathrm{t}}>$ & 0.037 & 0.037 & 0.037 \\
\hline$P_{\alpha}(\mathrm{MW})$ & 600 & 600 & 600 \\
\hline$P_{\mathrm{EC}}(\mathrm{MW})$ & 213 & 225 & 231 \\
\hline$P_{\text {brems }}(\mathrm{MW})$ & 37 & 37 & 37 \\
\hline$P_{\mathrm{EC}} P_{\alpha}(\%)$ & 34 & 36 & 37 \\
\hline$F_{\mathrm{rad}}\left(\mathrm{MW} / \mathrm{m}^{2}\right)$ & 0.20 & 0.45 & 0.83 \\
\hline
\end{tabular}

Due to the effects of reabsorption the net EC wave power radiated locally as well as the total EC power loss, as obtained from RAYTEC, are found to have a behaviour somewhat different from that of $\alpha$-particle heating and bremsstrahlung. In fact, as seen from Fig.5 and Table 2, $\left(\mathrm{d} P_{\mathrm{EC}} / \mathrm{d} V\right) /\left(\mathrm{d} P_{\alpha} / \mathrm{d} V\right)$ and $P_{\mathrm{EC}}$ do increase very slightly with increasing $B_{\mathrm{t} 0}$. This is because the combined effect of increasing $B_{\mathrm{t} 0}$ and correspondingly reducing the size of the plasma, so as does lowering wall reflection, tends to reduce the effective optical thickness 
of the plasma for EC waves. This effect is implicit in Trubnikov's scaling [7] for the total EC wave loss which, for the case at hand, yields $P_{\mathrm{EC}} \sim B_{\mathrm{t} 0}{ }^{1 / 6}$. The scaling is in agreement with the RAYTEC results with an accuracy around $1 \%$ for the parameter range considered.

Of course, for higher confining magnetic field the average radiative wall load $F_{\text {rad }}=\left(P_{\mathrm{EC}}+P_{\text {brems }}\right) / S$ with $S$ the first-wall surface, does strongly increase (see Table 2), approximately like $B_{\mathrm{t} 0}{ }^{17 / 6}$; therefore and because of the increasing compactness, high-field DEMO concepts, from a technological point of view, would be extremely demanding.

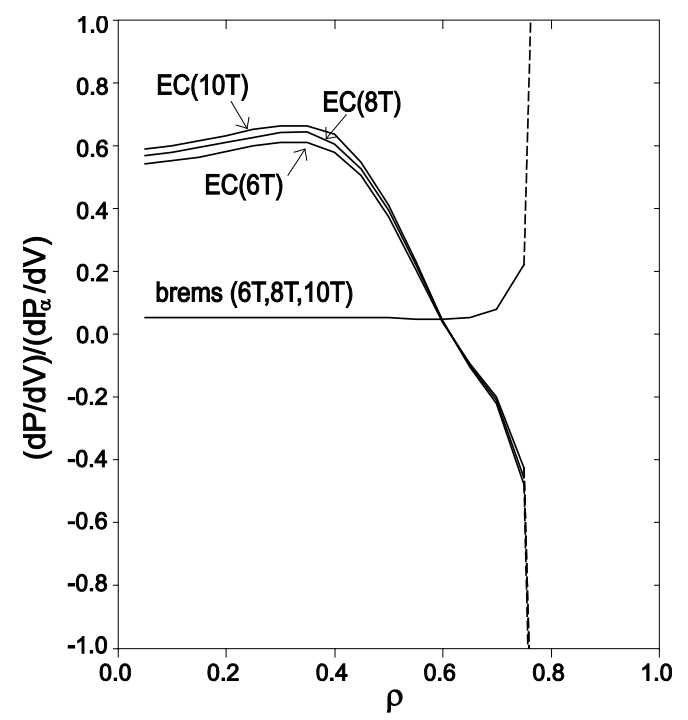

Figure 5. Radial profiles of net radiative EC wave $\left(\mathrm{d} P_{\mathrm{EC}} / \mathrm{d} V\right)$ and bremsstrahlung $\left(\mathrm{d} P_{\text {brems }} / \mathrm{d} V\right)$ power losses, normalised to the local $\alpha$-particle heating power density $\mathrm{d} P_{\alpha} / \mathrm{d} V$.

\section{Conclusions}

Using the RAYTEC code, a quantification of the importance of EC wave power transport and losses is given for a number of DEMO-like hot plasmas. In all cases these are significant and appreciably larger than bremsstrahlung effects. The calculated total EC wave power loss is well approximated by the LATF model [4]; also, its dependence on plasma parameters, wall reflection and the confining toroidal magnetic field, at constant fusion power, is well described by Trubnikov's scaling [7] for the parameter ranges considered.

\section{References}

1. D. J. Ward, Plasma Phys.Control.Fusion 52, 124033 (2010)

2. F. Albajar, M. Bornatici, G. Cortes, et al., Nucl. Fusion 45, 642 (2005)

3. F. Albajar, M. Bornatici, F. Engelmann, 2nd IAEA DEMO Programme Workshop, 17-20 December 2013, Vienna, Austria

4. F. Albajar, M. Bornatici, F.Engelmann, Nucl.Fusion 49, 115017 (2009)

5. B. Brunelli, Il Nuovo Cimento 55B, 264 (1980)
6. H. Zohm, C. Angioni, E. Fable, et al., Nucl.Fusion 53, 073019 (2013)

7. B. A. Trubnikov, in Review of Plasma Physics (M.A Leontovich, Ed.) Vol. 7, Consultants Bureau, New York (1979) 345

This publication reflects the views only of the author, and Fusion for Energy cannot be held responsible for any use which may be made of the information contained therein. 